\title{
CARACTERÍSTICAS GENERALES DE LOS ARTÍCULOS ORIGINALES INCLUIDOS EN LAS REVISIONES BIBLIOGRÁFICAS SOBRE SALUD E INMIGRACIÓN EN ESPAÑA
}

\author{
Elena Ronda-Pérez $(1,2,3)$, Gaby Ortiz-Barreda $(2,4)$, Cristina Hernando $(1,5,6)$, Carmen Vives- \\ Cases (2), Diana Gil-González (2) y Jordi Casabona (1,5,6,7,8,9 ).
}

(1) Subprograma Salud e Inmigración del CIBERESP. CIBER de Epidemiologia y Salud Pública.

(2) Grupo de Investigación en Salud Pública. Universidad de Alicante.

(3) Centro de investigación en salud laboral (CISAL). Universitat Pompeu Fabra.

(4) Departamento de Promoción de la Salud y Desarrollo-HEMIL Senteret-. Universidad de Bergen. Noruega.

(5) Centro de Estudios Epidemiológicos sobre las Infecciones de Transmisión Sexual y Sida de Cataluña (CEEISCAT).

(6) Departamento de Pediatría, Obstetricia y Ginecología y de Medicina Preventiva y de Salud Pública. Universidad Autónoma Barcelona. España.

(7) Instituto Catalán de Oncología (ICO).

(8) Agencia de Salud Pública de Catalunya (ASPC), Generalitat de Catalunya. España.

(9) Fundación Instituto de Investigación Germans Trias i Pujol (IGTP). Badalona. España.

\section{RESUMEN}

Fundamentos: La realidad socio-demográfica configurada en España a partir de la incorporación de la población inmigrante requiere analizar las necesidades y prioridades generadas por esta situación en todos los ámbitos, incluido el de la investigación en salud. El objetivo del presente estudio es conocer las características generales de los artículos incluidos en una revisión bibliográfica sobre este tema y realizada en el marco del Subprograma de Salud e Inmigración del CIBERESP

Métodos: Revisión bibliográfica de los artículos originales publicados en español o inglés en el periodo 1998-2012. Se seleccionaron artículos realizados en España y que cumplieran la definición de inmigrante de la Organización Internacional de Migraciones. La búsqueda bibliográfica se realizó en Medline y MEDES. Se analizó la distribución temporal de la producción y las características generales de los artículos mediante frecuencias absolutas y relativas.

Resultados: En la búsqueda inicial se identificaron 2.625 artículos (2.434 Medline y 191 Medes-MEDicina), finalmente se incluyeron los 311 que cumplían criterios de inclusión. La mayoría eran estudios epidemiológicos de diseño transversal realizados con datos primarios. En el $69 \%$ se comparó a la población inmigrante con la autóctona. En 217, ( $70 \%$ ) la temática principal fue la relacionada con enfermedades transmisibles. En $256(82 \%)$ el periodo producción fue entre 2004 y 2011. En $220(71 \%)$ el país de origen fue la forma más común de clasificación de la población inmigrante.

Conclusiones: Las enfermedades transmisibles fueron el principal objeto de investigación de los estudios desarrollados en España sobre salud de la población inmigrante. La mayoría de estudios incluyen a la población autóctona como grupo de comparación.

Palabras clave: Migración internacional. Salud. España. Desigualdades en la salud. Revisión.

\section{Correspondencia}

Elena Ronda

Carretera San Vicente del Raspeig, s/n

03690 Alicante

Elena.Ronda@ua.es

\section{ABSTRACT}

\section{General Characteristics of the Original Articles Included in the Scoping Review on Health and Immigration in Spain}

Background: The new socio-demographic reality that came about with the incorporation of the immigrant population in Spain requires an analysis of the needs and priorities generated by this situation in all areas, including research in health. The objective of this study is to determine the general characteristics of a group of articles included in a literature review on the subject, carried out within the framework of the CIBERSP Subprogram on Migration and Health.

Methods: Scoping Review of the literature published in the period 1998-2012. Articles in Spanish or English developed in Spain and that fulfil the definition of immigrant from the International Organization for Migration were selected. The literature search was performed in Medline and MEDES. The temporal distribution of the production and main characteristics of the articles are described trough absolute and relative frequencies.

Results: The initial search identified 2.625 articles (Medline 2434; 191 Medes-MEDicina) 311 were including finally. Most epidemiological studies are cross-sectional design with primary data. $69 \%$ compared with native population. The main theme has been associated with infectious diseases $(n=217,70 \%)$. The period of maximum production is between 2004 and $2011(\mathrm{n}=256,82 \%)$. The country of origin is the most common way of classifying immigrants $(n=220,71 \%)$.

Conclusions: The epidemiology of infectious prevails as the main theme of the studies performed in Spain about the health of the immigrant population. Most of the studies include native population as a comparison group.

Keyword: Emigrants and Immigrants. Emigration and Immigration. Transients and Migrants. Ethnic Groups. Spain. Health. Health care. Disparities. Review. 


\section{INTRODUCCIÓN}

Tanto por sus implicaciones sociales como sanitarias, la inmigración es un tema de gran interés y debate en el campo de la salud pública ${ }^{1-3}$. En el caso de España, este fenómeno empezó a hacerse visible desde los últimos años de la pasada centuria cuando el saldo migratorio cambió de signo. Este cambio caracterizado por lo que se conoce como la "singularidad de España" implicó que durante un periodo de 8 años (desde 2000 a 2008) la población migrante empadronada creció en más de 4 millones de personas. Ningún país de la Unión Europa alcanzó la magnitud migratoria de España. La llegada de inmigrantes extranjeros a España se presentó de forma repentina y acelerada, en poco tiempo se alcanzaron cifras de tal magnitud que situaron a España al mismo nivel que otros países de acogida con mucha mayor tradición migratoria como Reino Unido o Alemania ${ }^{4}$. Así, en 1981 el total de la población inmigrante extranjera en España era de 625.907 personas, un $1,7 \%$ de la población total. Dieciocho años después, en 1999, la población extranjera era de 748.953 y el porcentaje sobre la población muy similar, el 1,9\%. Sin embargo, en los cinco años siguientes el ritmo de entrada fue tal que se alcanzó en 2005 la cifra de 3.691 .547 personas extranjeras, es decir un $8,4 \%$ del total de la población ${ }^{4}$. En los años siguientes el aumento de la población migrada continuó, tal como refleja el último censo del 1 de noviembre de 2011 que contabiliza 6.307.277 personas extranjeras, el 13,5\% de la población total, casi el triple del censo previo de 2001 (2.172.201 personas, el $5,3 \%$ de la población $)^{4}$. La cifra disponible más reciente es el padrón del 1 de enero de 2013, que, a pesar del ligero descenso de la población migrada causado por la crisis económica que vive el país, contabiliza 5.520 .133 personas extranjeras, el $11,73 \%$ del total de la población (47.059.533 personas) ${ }^{5}$. Así mismo, también destacaba la elevada proporción de población inmigrante extranjera en situación irregular a su lle$\operatorname{gada}^{6,7}$.

Previamente a 1998, el crecimiento de la población migrada estuvo protagonizado por población africana, seguida a mucha distancia por población asiática y latinoamericana 5 . Sin embargo, desde 1998 el gran incremento de la población inmigrante fue protagonizado por la afluencia de población procedente de América Latina y Europa del Este ${ }^{5}$.

El proceso migratorio conlleva habitualmente cierto grado de desestructuración del entorno de las personas. Los colectivos inmigrantes más vulnerables generalmente presentan, además de los riesgos asociados a las condiciones del viaje, un escaso conocimiento de los principios de promoción o protección de la salud. Se enfrentan a una cultura sanitaria y a un sistema sanitario desconocidos y con frecuencia no comprenden la organización asistencial. En el lugar de destino pueden añadirse dificultades como el desarraigo, la estigmatización, la falta de apoyo social y familiar, la movilidad geográfica, la precariedad laboral, la discriminación y las dificultades lingüísticas y legales, entre otras. Por todo ello, la movilidad de la población puede tener repercusiones en la salud de los inmigrantes, de la sociedad de origen, de la sociedad receptora $\mathrm{y}$, finalmente, de la salud pública global ${ }^{2,8}$.

La realidad socio-demográfica configurada en España a partir de la incorporación de la población inmigrante en su tejido social requiere analizar las necesidades y prioridades generadas por esta situación en todos los ámbitos, incluido el de la investigación en salud, con especial énfasis en el impacto en la salud producido durante los primeros años de asentamiento de la población inmigrante. Por 
este motivo el CIBER de Salud Pública (CIBERESP) creó en 2012 el Subprograma de Salud e Inmigración (SIIS), el cual tienen como propósito estudiar el estado de salud de la población inmigrante en España, así como sus determinantes biológicos, conductuales y estructurales. El SIIS está adscrito al Programa de determinantes para la Adquisición y Propagación de Enfermedades Transmisibles (DAPET) y actualmente cuenta con 11 grupos del CIBERESP y 2 de externos. El SPIIS se ha organizado alrededor de los ámbitos y líneas de trabajo que constan en la tabla 1 .

Como una de sus primeras actividades y con la finalidad de sintetizar el conocimiento científico sobre el estado de salud y la utilización de servicios sanitarios por parte de la población inmigrante en España en el periodo entre 1998 y 2012 y sus determinantes, así como identificar vacíos de conocimiento que abordar en futuras investigaciones el Subprograma realizó una revisión de la literatura para cada una de las líneas de trabajo definidas. Los resultados de cada una de las temáticas analizadas serán incluidos en los correspondientes artículos específicos.
El objetivo del presente estudio es conocer las características generales de los artículos incluidos en estas revisiones bibliográficas.

\section{MATERIAL Y MÉTODOS}

Diseño. Se realizó una revisión de artículos originales publicados que abordaban el estado de salud de la población inmigrante en España. Para ello, se utilizó la metodología de revisión bibliográfica o Scoping Review (SR) ${ }^{9}$. La búsqueda y selección de los artículos se estructuró de acuerdo a las líneas de trabajo de los grupos de investigación que componen el Subprograma de Salud e Inmigración del CIBERESP (tabla 1).

Estrategia de búsqueda e identificación de los artículos. Se consultaron las bases de datos de Medline a través de Pubmed y MEDES-MEDicina en español debido a su mayor visibilidad en el área sanitaria a nivel internacional y nacional. Se seleccionaron todos los artículos publicados entre enero de 1998 y diciembre de 2012. La decisión de limitar la búsqueda al período establecido se basó en la intención de explorar toda la producción científica

Tabla 1

Líneas y grupos de investigación del Subprograma de Salud e Inmigración del Ciber en Epidemiología y Salud Pública (CIBERESP)

\begin{tabular}{|c|c|}
\hline LÍNEAS DE INVESTIGACIÓN & GRUPOS \\
\hline \multirow{3}{*}{$\begin{array}{c}\text { DETERMINANTES } \\
\text { CONTEXTUALES }\end{array}$} & Desigualdades en salud \\
\hline & Salud laboral \\
\hline & Políticas, sistemas y servicios de salud \\
\hline \multirow{3}{*}{$\begin{array}{c}\text { ENFERMEDADES } \\
\text { NO TRANSMISIBLES }\end{array}$} & Salud mental y drogodependencias \\
\hline & Prevención de cáncer \\
\hline & Nutrición y factores de riesgo cardiovascular \\
\hline \multirow{6}{*}{$\begin{array}{l}\text { ENFERMEDADES } \\
\text { TRANSMISIBLES }\end{array}$} & VIH y otras infecciones de transmisión sexual \\
\hline & Tuberculosis y enfermedades inmunoprevenibles \\
\hline & Enfermedades importadas \\
\hline & Dinámica poblacional de las resistencias a antibióticos \\
\hline & Epidemiologías molecular y genómica \\
\hline & Papel de los agentes infecciosos en las enfermedades no transmisibles \\
\hline
\end{tabular}


generada sobre este tema desde el inicio del incremento progresivo de la llegada de población inmigrante en España. El término limitador por contenido fue la especie humana. En cuanto al idioma, se seleccionaron las publicaciones escritas en español o en inglés.

Se utilizaron los términos del Medical Subject Headings [MeSH]. Se desarrollaron 3 filtros temáticos: a) Filtro sobre el estado de salud/enfermedad: MeSH y palabras clave proporcionados por los respectivos grupos de investigación del Subprograma de Salud e Inmigración (anexo 1); b) Filtro inmigración: Emigrants and Immigrants, Emigration and Immigration, Transients and Migrants y Ethnic Groups; c) Filtro país: se utilizó el desarrollado por Valderas J, y colaboradores ${ }^{10}$. En el caso de MEDES-MEDicina en español que no cuenta con tesauros, se utilizó únicamente las siguientes palabras clave como palabras naturales. Concretamente, se realizó la siguiente combinación: "migración" OR "migrantes" OR "migrante" OR "inmigración", "inmigrantes" OR “inmigrante”. No se utilizó ningún límite de fecha. La búsqueda se limitó por tipo de publicación (artículos originales).

Criterios de selección de estudios y extracción de la información. El proceso de la identificación de los artículos correspondientes a los grupos de investigación se ejecutó por una persona (GO) la cual remitió las referencias localizadas en la búsqueda a los grupos correspondientes que eran los que decidían si la referencia se ajustaba al objetivo del estudio. Para el desarrollo de esta SR, se utilizó la definición de migrante otorgada por la Organización Internacional para las Migraciones (OIM) ${ }^{11}$ : “...personas, y los miembros de la familia, que deciden mudarse a otro país para mejorar sus condiciones materiales o sociales y mejorar la perspectiva para sí o para su familia”.
Posteriormente a la identificación de los estudios, los responsables de los grupos de investigación procedieron a la selección inicial de sus artículos basándose en la lectura de los títulos y resúmenes. Los criterios de inclusión fueron los siguientes:

1) Estudios originales cualitativos y cuantitativos, publicados en revistas científicas sobre: a) Estado de salud, b) Programas y/o actividades preventivas, c) Determinantes sociales, laborales, culturales y/o económicos de salud, y d) Acceso y uso de los servicios sanitarios en la población inmigrante, que habían sido desarrollados en España. 2) Estudios que incluían población inmigrante procedentes de Centro y Sud-América, África, Asia y Europa del Este desarrollados en España.3) Estudios que aunque no especificas en la nacionalidad o país de origen de la población, incluían las categorías de “inmigrantes", “extranjeros” o análogos.

Se excluyeron los estudios que abordaban otros tópicos no relacionados con la temática, artículos sin resumen disponible, repetido en otras bases de datos, así como, los editoriales de revistas científicas y los artículos de revisión sobre el tema. También los artículos que se centraban en población inmigrante procedente de Norteamérica: Canadá y Estados Unidos de América, Oceanía, o Europa Occidental (Alemania, Andorra, Austria, Bélgica, Ciudad del Vaticano, Croacia, Dinamarca, Grecia, Finlandia, Francia, Holanda, Irlanda, Islandia, Italia, Liechtenstein, Luxemburgo, Malta, Mónaco, Noruega, Portugal, Reino Unido, San Marino, Suecia y Suiza).

Posteriormente se revisaron a texto completo para comprobar que cumplían los criterios de inclusión. El proceso se presenta en la figura 1.

Para la extracción y síntesis del contenido de los artículos incluidos se diseñó 


\section{Figura 1}

Proceso de selección de artículos en la revisión bibliográfica sobre el estado de salud y sus determinantes en la población inmigrante en España (1998-2012)

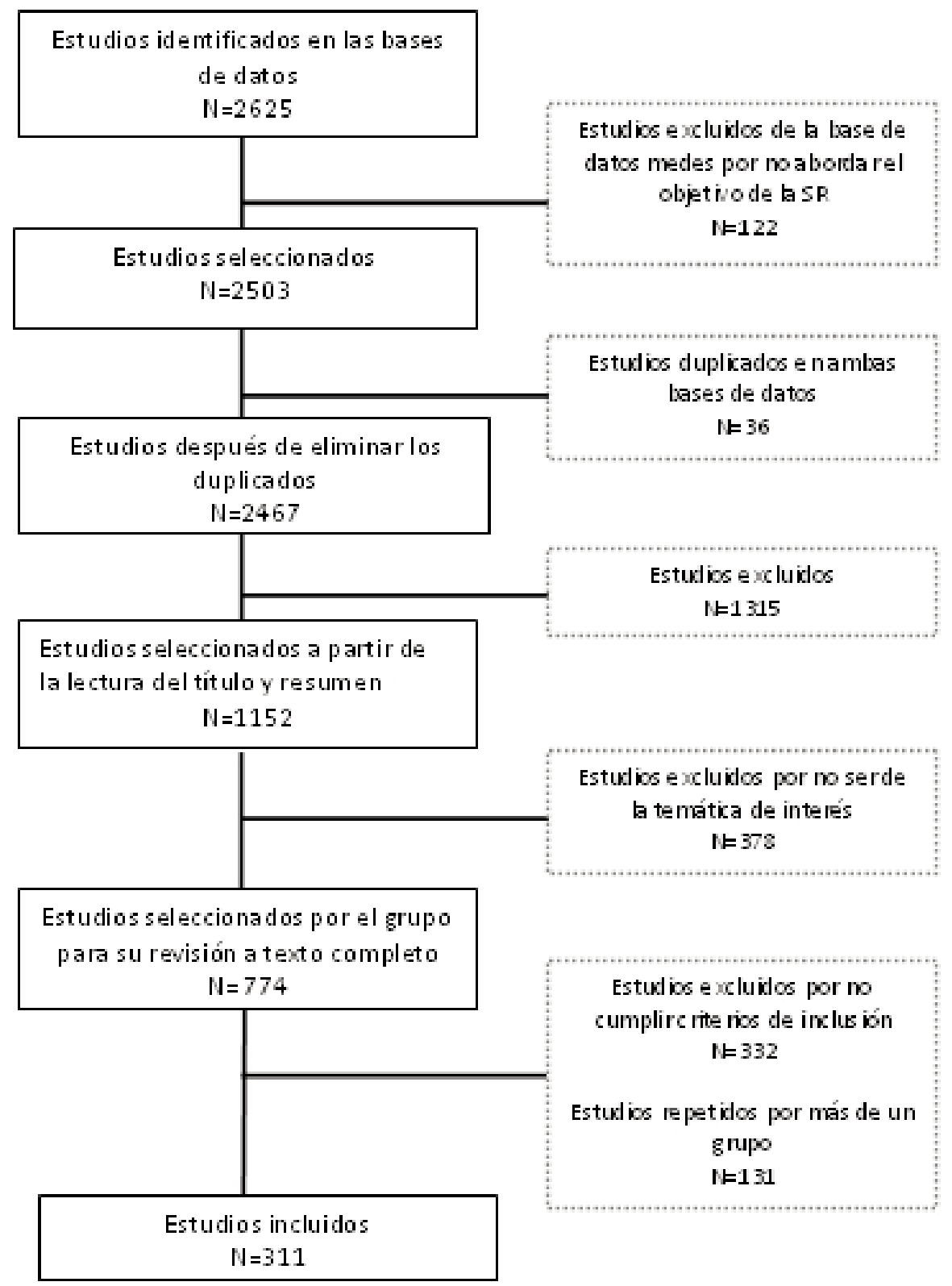


una herramienta de recogida de información con apartados relativos a las características generales de los artículos según los apartados de objetivos, metodología, principales resultados y conclusiones a partir de la lectura completa del artículo. Posteriormente, se digitalizaron sus respuestas utilizando Google DRIVE. La cumplimentación del registro de recogida de información fue realizada por cada grupo, a través del vínculo informático generado por este sistema los resultados extraídos eran grabados automáticamente en la hoja de cálculo Excel.

\section{RESULTADOS}

En la búsqueda inicial se identificó un total de 2.625 referencias. Después de su revisión, se excluyeron $2.183(83,1 \%)$ por tratarse de estudios no relacionados con el objetivo de la investigación y por no cumplir los criterios de inclusión (figura 1). Al final quedaron 442 (16,8\%) correspondientes a 11 grupos de investigación. 131 estudios fueron compartidos por varios grupos, por lo que el número final de artículos fue de 311 .

Evolución temporal de la publicación científica. En general, la producción científica sobre Inmigración y Salud en España mostró una tendencia ascendente durante el periodo de $2004(\mathrm{n}=17)$ a 2012 $(\mathrm{n}=29)$. Con respecto a la evolución anual global, se observó que este incremento se produjo principalmente en $2009(\mathrm{n}=70$; $22,5 \%), 2011(\mathrm{n}=50 ; 16,0 \%)$ y 2010 $(\mathrm{n}=40 ; 12,8 \%)$. Otros aumentos se identificaron en los años de 2012 y 2008 ( $n=29$; $n=27$ ). Diferenciado por las tres líneas de investigación, se observó que el mayor número de artículos identificados correspondió a la línea de enfermedades trasmisibles $(n=217 ; 69,7 \%)$ seguida de la línea de determinantes contextuales $(n=58$; $18,7 \%$ ) y las enfermedades no transmisibles $(n=36 ; 11,6 \%)$. En el año 2009 se observó un incremento notorio en la línea de enfermedades transmisibles $(n=43)$, así

Figura 2

Distribución temporal de la producción científica sobre salud e inmigración en España (1998-2012) según las diferentes líneas de investigación $(n=311)$

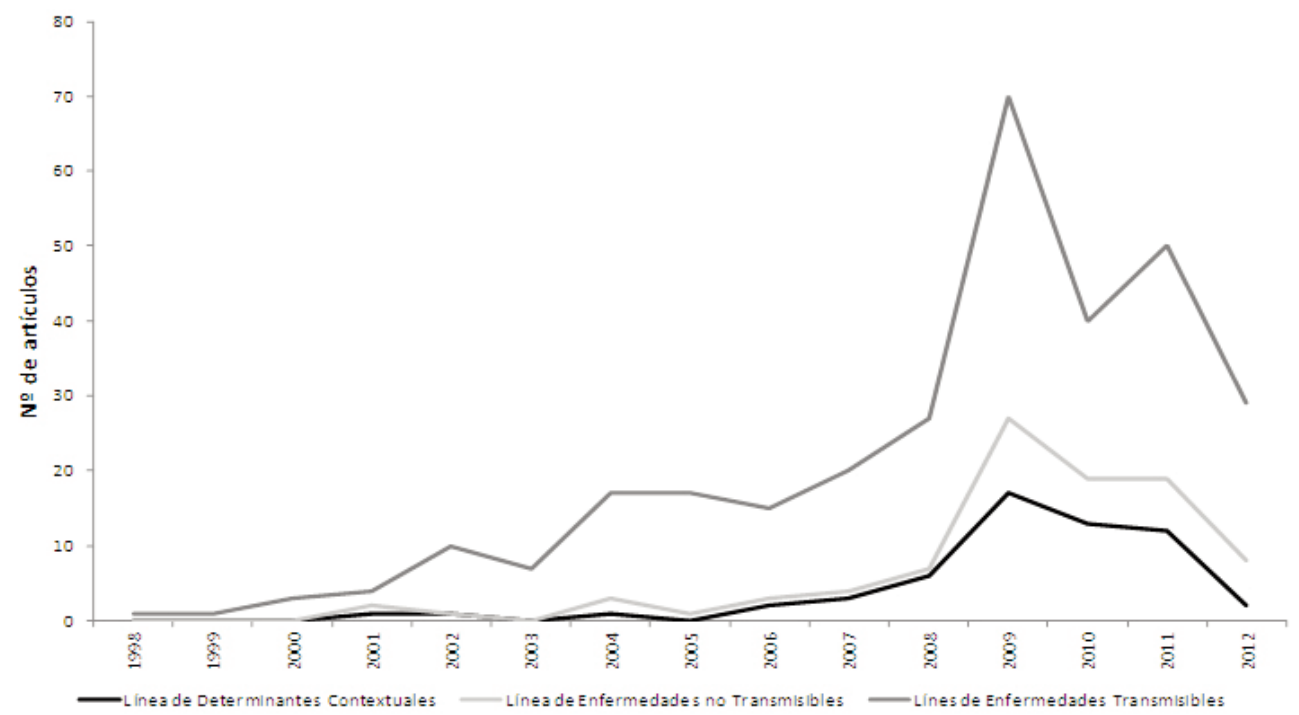




\section{Tabla 2}

Características de los artículos incluidos en la revisión bibliográfica sobre el estado de salud y sus determinantes en la población inmigrante en España (1998-2012) $(n=311)$

\begin{tabular}{|l|c|c|}
\hline \multirow{2}{*}{ Diseño de investigación } & \multicolumn{2}{|c|}{ Artículos } \\
\cline { 2 - 3 } & $\mathrm{n}$ & $\%$ \\
\hline Epidemiológicos & 283 & 91,0 \\
\hline Cualitativa & 15 & 4,8 \\
\hline Ciencias básicas & 13 & 4,2 \\
\hline Diseño epidemiológicos & 158 & 55,8 \\
\hline Transversal & 9 & 3,2 \\
\hline Casos-Controles & 79 & 28,0 \\
\hline Estudios de cohorte & 5 & 1,7 \\
\hline Estudios Clínicos & 6 & 2,1 \\
\hline Estudios Ecológicos & 26 & 9,1 \\
\hline Otros (series de casos, estudios de mortalidad,...) & \multicolumn{3}{|l}{} \\
\hline Técnicas cualitativas & 8 & 53,3 \\
\hline Grupos focales/entrevistas semiestructuradas & 8 & 192 \\
\hline Entrevistas semiestructuradas & 7 & 46,7 \\
\hline Fuentes de datos & 192 & 61,7 \\
\hline Primarias & 99 & 31,8 \\
\hline Secundarias & 20 & 6,5 \\
\hline Ambos & 262 & 84,2 \\
\hline Población incluida en estudios epidemiológicos \\
\hline Solo inmigrantes & 62,4 \\
\hline Inmigrantes/autóctonos & 27,9 \\
\hline Consideración legal de la población inmigrante \\
\hline Si & 49 & 15,8 \\
\hline No & 20 \\
\hline
\end{tabular}

como en la línea de determinantes contextuales $(n=17)$ y en la de enfermedades no transmisibles $(\mathrm{n}=10)$ (figura 2).

Características generales de los estudios. De acuerdo con los artículos incluidos por cada grupo de investigación participante $(n=442)$, se observó que un total de 102 pertenecían al grupo de el papel de los agentes infecciosos en las enfermedades no transmisibles, 95 a enfermedades importadas, 61 a dinámica poblacional de las resistencias a antibióticos, 47 a epidemiología molecular y genómica, 29 a VIH y otras infecciones de transmisión sexual.
En otros grupos relacionados con el estudio de los determinantes contextuales se identificó un total de 27 en el grupo de desigualdades en salud, 20 en salud laboral y 21 en el grupo de políticas, sistemas y servicios de salud. En los grupos relacionados a las enfermedades no transmisibles se incluyeron 19 artículos por el grupo de salud mental y drogodependencias, 5 prevención del cáncer y 16 por el grupo de nutrición y riesgo cardiovascular.

Del total de los 311 estudios incluidos (excluyendo los repetidos por varios grupos) $15(4,8 \%)$ utilizaron metodología cualitativa. En estos estudios los grupos focales y las entrevistas semi-estructuradas fueron las técnicas más empleadas ( 8 ; $53,3 \%) .283$ (91,0\%) estudios presentaban diseños epidemiológicos. 158 (55,8\%) estudios tenían un diseño transversal y 79 $(28,0 \%)$ de cohorte. Sólo 9 (3,2\%) estudios eran de casos y controles (tabla 2).

En 192 (61,7\%) artículos las fuentes primarias fueron las más utilizadas, en 99 $(31,8 \%)$ eran fuentes secundarias. Con respecto a la población incluida 196 $(69,3 \%)$ estudios compararon a la población inmigrante con la población autóctona y en $87(30,7 \%)$ sólo se incluyó como participantes a inmigrantes. En 49 artículos se consideró el estatus legal o ilegal de la población inmigrante (definido como disponer o no de permiso de trabajo y/o residencia) (tabla 2 ).

En el $71 \%$ el status migratorio se definió según el país de origen/nacimiento y en $9 \%$ con la nacionalidad. En 47 artículos se utilizó la combinación del país de origen/nacimiento, nacionalidad con el tiempo de residencia (figura 3 ).

Los estudios que identificaron países concretos de procedencia incluyeron inmigrantes de Ecuador, Colombia, Bolivia, Paraguay, Perú, Guinea Ecuatorial, Argelia, Marruecos y Rumania. 


\section{Figura 3}

Definición de la variable inmigración utilizada en los estudios sobre salud e inmigración en España (1998-2012) en relación a la temática

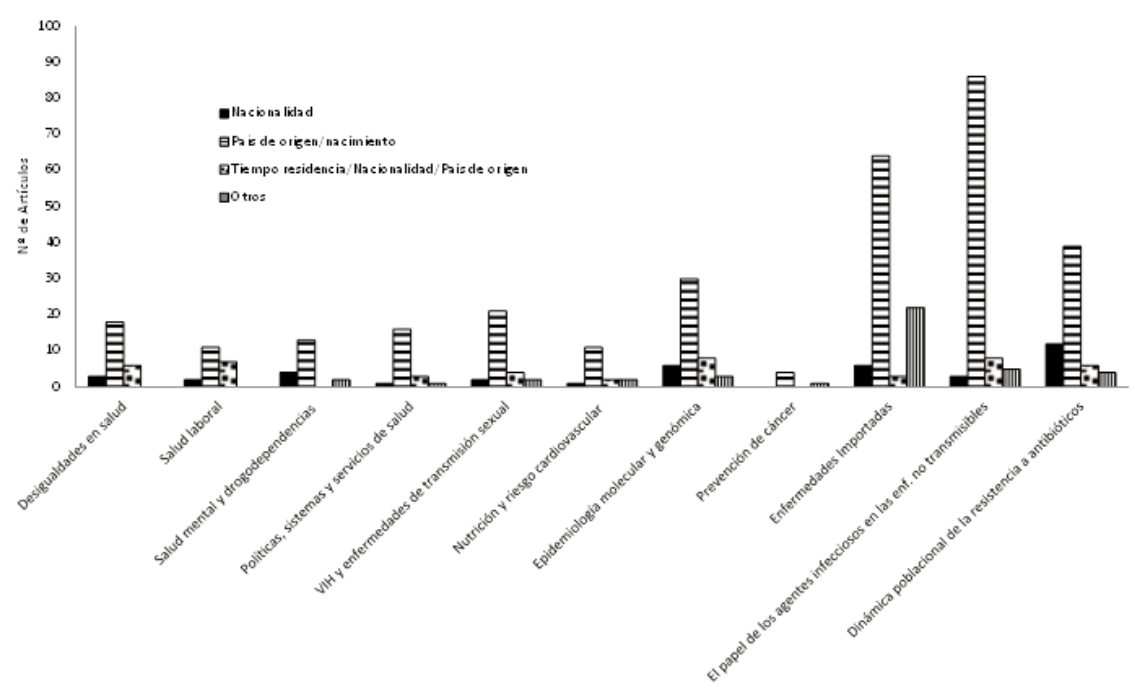

\section{DISCUSIÓN}

Los resultados ponen de manifiesto que en paralelo al cambio demográfico por el proceso demográfico acontecido en España en los últimos años del siglo XX y comienzos del XXI se generó un incremento en investigaciones que tuvieron como finalidad profundizar en el binomio inmigración y salud. En 1998, sólo se encuentra una publicación, cinco años más tarde este número ascendía a 60 . Estos resultados coinciden con los hallazgos previos de otra revisión sobre esta temática ${ }^{12}$.

De una manera muy general, se pueden diferenciar dos tipos de investigaciones. Por una parte, las que tuvieron como objetivo describir el patrón epidemiológico de determinados procesos de salud ${ }^{13}$ y de diferentes determinantes ${ }^{14,15}$. Por otra, las que buscaban adquirir un mayor conocimiento de los procesos que pueden estar implicados en la relación entre proceso migratorio y el desarrollo de ciertos procesos de salud ${ }^{16-18}$.
La mayor parte de artículos incluidos se basaban en investigaciones epidemiológicas. Sobre todo de diseño trasversal. En este sentido, cabe señalar que recientemente se han desarrollado modelos teóricos para el estudio de la salud en la población inmigrante que aconsejan un enfoque amplio que incorpore una perspectiva del curso de vida $^{19,20}$. En España, como en el resto de países en general, los estudios longitudinales realizados específicamente en población inmigrante hasta el momento son escasos y presentan dificultades y retos propios. Éste debería ser un nuevo campo para la investigación de la salud en la población migrada.

Comparando con la literatura internacional contrasta el alto porcentaje de estudios con datos primarios ${ }^{1}$. Esto, puede reflejar, sobre todo en los primeros años, la ausencia de información en registros oficiales de la inclusión de algún tipo de variable que pudiera identificar el estatus migratorio ${ }^{21}$ que con el paso del tiempo ha ido incorporándose. 
En la literatura revisada el modo de identificación más frecuente de la población inmigrante es el país de origen/nacimiento, sin embargo, este hecho contrasta con el uso mayoritario de la variable nacionalidad en otras fuentes de información, como los sistemas de vigilancia de VIH/Sida en Europa, en los que únicamente Bélgica, Dinamarca y España recogen "país de origen" 22 .

El enfoque flexible que permite realizar esta metodología en la selección y síntesis de la literatura existente la hace especialmente adecuada para los fines de esta revisión sobre el estado de salud y sus determinantes en la población inmigrante en España. No obstante, hay que mencionar algunas limitaciones, como el hecho de que las palabras clave utilizadas fueron las facilitadas dependiendo del interés de los respectivos grupos incluidos en el subprograma, con lo cual algunas áreas, como es el caso de pediatría y ginecología y obstetricia, por ejemplo, no se han visto reflejadas. No obstante, dada la transversalidad de los temas sí que se han localizado algunos artículos de estas materias. Aunque las bases de datos consultadas albergan un volumen importante de información en ciencias de la salud, probablemente exista información científica sobre el tema objeto de estudio que no haya sido identificada en ellas, y sobre todo de forma específica en la literatura gris. En este sentido, es posible que pudiera obtenerse más información a través de informes y documentos de organismos públicos u organizaciones no gubernamentales dedicadas al estudio de la inmigración y salud en España.

En conclusión, a pesar de que la investigación de salud pública sobre migración y salud ha aumentado en los últimos años paralelamente al aumento de esta población, el volumen de publicaciones científicas parece aún poco extenso -predominando las enfermedades transmisibles-y heterogéneo en relación a las metodologías y terminologías usadas. Teniendo en cuenta el actual escenario demográfico con una importante población migrada ya establecida en el país, la complejidad de la relación entre migración y salud y el debate abierto sobre la misma en la sociedad española ${ }^{3}$, es necesaria una mayor y más transversal investigación sobre migración y salud, así como una mayor coordinación entre grupos investigadores. En particular, sería importante contar con estudios longitudinales que puedan incorporar el impacto del tiempo de residencia en el país y que los sistemas de información sistematicen y armonicen las variables referentes al estatus migratorio. El subprograma de Salud e Inmigración de CIBERESP, con la colaboración de grupos de otros CIBERS y redes puede contribuir a ello.

\section{BIBLIOGRAFÍA}

1. Foets M. Improving the quality of research into the health of migrant and ethnic groups. Int J Public Health. 2011;56:455-6.

2. Oliva J, Perez G. Inmigación y salud. Gac Sanit. 2009;23 Suppl 1:1-3.

3. Bhopal RS. Research agenda for tackling inequalities related to migration and ethnicity in Europe. J Public Health 2012;34:167-73.

4. Maruri Antuñano I, Claramunt, Ochando C, Guillen Soler Á. Inmigración, mercado de trabajo y Seguridad Social: evidencia empírica española e implicaciones de política económica. Revista del Ministerio de Trabajo e Inmigración 2002;81:17-39

5. Instituto Municipal de Estadística. Padrón Municipal de Habitantes. [Citado 10-1-2014]. Disponible en: http://www.ine.es/jaxi/menu.do?type=pcaxis\&path $=/ \mathrm{t}$ 20/e245/\&file=inebase.

6. Pajares M. Inmigración y Mercado de Trabajo. Informe 2008. Madrid: Ministerio de Trabajo e Inmigración; 2008.

7. Punto de Contacto Nacional de España de la Red Europea de Migraciones. Medidas Prácticas para la Reducción de la Inmigración Irregular. Madrid: Observatorio Permanente de la inmigración; 2011.

8. Bhopal R, Rafnsson S. Global inequalities in assessment of migrant and ethnic variations in health. $\mathrm{J} \mathrm{Pu}-$ blic Health. 2012;126:241-4. 
9. Arksey H, O'Malley L: Scoping studies: towards a methodological framework. Int J Soc Res Methodol. 2005, 8:19-32

10. Valderas JM, Mendivil J, Parada A, Losada-Yanez M, Alonso J. Construcción de un filtro geográfico para la identificación en PubMed de estudios realizados en España. Rev Esp Cardiol. 2006;59:1244-51

11. Perruchoud R, Redpath-Cross J. Glossary on Migration. 2nd ed: Ginebra: International Organization for Migration; 2011.

12. Berra S, Elorza-Ricart J. Salud y uso de servicios en los sistemas sanitarios en población autóctona e inmigrante de España. Madrid: Plan de Calidad para el Sistema Nacional de Salud Ministerio de Ciencia e Innovación. Barcelona: Agència d'Avaluació de Tecnologia i Recerca Mèdiques de Cataluña; 2009.

13. Gutiérrez M, Tajada P, Alvarez A, et al. Prevalence of HIV-1 non-B subtypes, syphilis, HTLV, and hepatitis $\mathrm{B}$ and $\mathrm{C}$ viruses among immigrant sex workers in Madrid, Spain. J Med Virol. 2004;74:521-7.

14. Agudelo-Suarez AA, Ronda-Pérez E, Gil-González D, Vives-Cases C, García AM, García-Benavides $\mathrm{F}$, et al. Proceso migratorio, condiciones laborales y salud en trabajadores inmigrantes en España (proyecto ITSAL). Gac Sanit. 2009;23 Suppl 1:115-21

15. Prado C, Roville-Sausse F, Marrodan D, Muñoz B, Fernández del Olmo R, Calabria V. Situación somatofisiológica y nutricional de los jóvenes inmigrantes en España. Variación según género y procedencia. Arch Latinoam Nut. 2011;61:367-75.

16. Regidor E, Ronda E, Pascual C, Martínez D, Calle ME, Domínguez V. Mortalidad por enfermedades cardiovasculares en inmigrantes residentes en la Comunidad de Madrid. Med Clínic. 2009;132:621-4.

17. Salinero-Fort MA, Jimenez-Garcia R, del OteroSanz L, del Otero-Sanz L, de Burgos-Lunar C, ChicoMoraleja RM, et et al. Self-reported health status in primary health care: the influence of immigration and other associated factors. PLoS One. 2012;7:e38462.

18. Malmusi D, Borrell C, Benach J. Migration-related health inequalities: showing the complex interactions between gender, social class and place of origin. Soc Sci Med. 2010;71:1610-9.

19. Spallek J, Zeeb H, Razum O. What do we have to know from migrants' past exposures to understand their health status? a life course approach. Emerg Themes Epidemiol. 2011;8:6.
20. Zimmerman C, Kiss L, Hossain M. Migration and health: a framework for 21 st century policy-making. PLoS Med. 2011;8:e1001034.

21. Levecque K, Benavides FG, Ronda E, Van ronen R. Use of existing health information systems for migrant health research in Europe: challenges and opportunities. In: Ingleby D, Krasnik A, Lorant V, et al., eds. Health inequalities and risk factors among migrants and ethnic minorities. Antwerp - Apeldoorn: Garant Publishers; 2012.p.53-68.

22. Del Amo J, Broring G, Hamers FF, et al. Monitoring HIV/AIDS in Europe's migrant communities and ethnic minorities. AID.S 2004;18:1867-73.

23. Carme Borrell, Davide Malmusi. La investigación sobre los determinantes sociales y las desigualdades en salud: evidencias para la salud en todas las políticas. Gac Sanit. 2010;24 (Supple 1):101-08. 


\section{Anexo 1}

Relación de palabras clave utilizadas en la búsqueda de artículos (1998-2012)

\begin{tabular}{|c|c|}
\hline Líneas de investigación & Palabras clave \\
\hline $\begin{array}{l}\text { Determinantes } \\
\text { contextuales }\end{array}$ & $\begin{array}{l}\text { "Work", "Work Schedule Tolerance", "Employment", "Job Satisfaction", "Acci- } \\
\text { dents, Occupational", "Occupational Diseases", "Occupational Health", "Quality of } \\
\text { Health Care", "Health Services Accessibility", "Health Services Needs and De- } \\
\text { mand", "Healthcare Disparities", "Needs Assessment", "Health Policy", access*,ne- } \\
\text { eds, demand, barriers, health service*, health care, healthcare. Y filtro sobre des- } \\
\text { igualdades en salud construido por Borrell y Malmusi'22. }\end{array}$ \\
\hline $\begin{array}{l}\text { Enfermedades } \\
\text { no transmisibles }\end{array}$ & $\begin{array}{l}\text { Antisocial Personality Disorder, Paranoid Personality Disorder, Schizoid Persona- } \\
\text { lity Disorder, Schizotypal Personality Disorder, Borderline Personality Disorder, } \\
\text { Histrionic Personality Disorder, Personality Disorders, Dependent Personality Di- } \\
\text { sorder, Compulsive Personality Disorder, Mental health, : diet, physical activity, } \\
\text { motor Activity tobacco, smoking, obesity, hypertension, dyslipidemias, diabetes } \\
\text { Mellitus, metabolic syndrome X,"Early Detection of Cancer", "Mass Screening", } \\
\text { "Neoplasms", "Breast Neoplasms", "Gastrointestinal Neoplasms", "Genital Neo- } \\
\text { plasms", "Uterine Cervical Neoplasms", "Cancer Care Facilities", "Smoking Cessa- } \\
\text { tion", "Smoking/prevention and control", "prevention and control" [Subheading], } \\
\text { tumors, cancer, cancers, neoplasm, breast cancer, Cervical cancer, colon cancer, } \\
\text { prostatic cancer, tobacco smoking, cancer screening. }\end{array}$ \\
\hline Enfermedades trasmisibles & 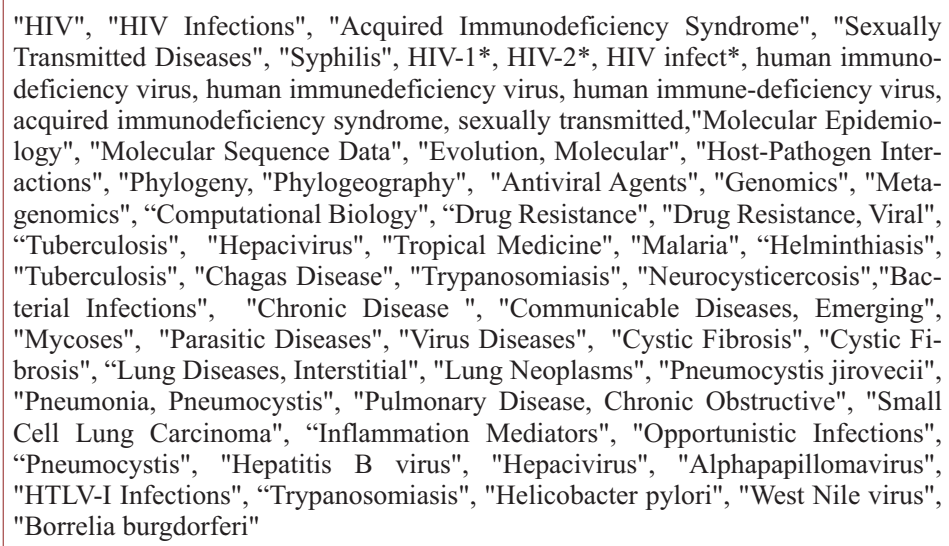 \\
\hline
\end{tabular}

